\title{
The effects of brief variable foreperiods on simple reaction time
}

\author{
DONALD J. POLZELLA, ERIC G. RAMSEY, and SAMUEL M. BOWER \\ University of Dayton, Dayton, Ohio
}

\begin{abstract}
Highly practiced subjects responded to a visual stimulus that was presented with a probability of 1.00 (no catch trials) or $\mathbf{0 . 9 0}$ (catch trials) after one of five randomly determined brief foreperiods $(300,400,500,600$, or $700 \mathrm{msec})$. Mean reaction time decreased linearly under the no catch-trials condition and curvilinearly under the catch-trials condition, as a function of increasing foreperiod duration. The results were consistent with the hypothesis that reaction time covaries with the momentary probability of occurrence of the response signal. Specifically, an increase in this momentary probability leads to an increase in the subject's readiness to detect the signal. Because performance was virtually errorless, it appears that any concomitant tendency to anticipate the signal can be successfully inhibited.
\end{abstract}

In a typical simple reaction-time task, a subject is required to respond to a signal as quickly as possible. The presentation of the response signal (RS) may be preceded by a warning signal (WS), which is provided in order to alert the subject to the subsequent occurence of the RS. The interval between the WS and the RS is known as the warning interval or foreperiod (FP). In cases where the FP varies from trial to trial, one can plot a function that relates the length of the FP to the reaction time (RT) associated with the RS. Many variables affect the shape of this function, including the duration, regularity, range, and distribution of the FP, and the modality, intensity, probability, and duration of the WS and RS. A comprehensive review of these effects can be found in Niemi and Naatanen (1981).

When the warning interval is a rectangularly distributed random variable, the function tends to decrease monotonically; that is, as the FP lengthens, the RT decreases. Nickerson (1965) proposed an expectancy hypothesis to account for the general shape of this function. According to this hypothesis, RTs will be short if expectancy is high, and vice versa. If a subject even roughly knows the shortest and longest FPs and knows that the duration of the FP on any given trial is equally likely to be anywhere between these limits, then the imminent occurrence of the RS will seem more and more likely as the waiting time following the WS increases. As FP lengthens, therefore, expectancy will rise and RT will decrease.

Preliminary reports of these findings were presented at the 25th Annual Meeting of the Psychonomic Society, San Antonio, TX, in November 1984, and at the 27th Annual Meeting of the Psychonomic Society, New Orleans, LA, in November 1986. This report is based in part on a thesis submitted by E. G. Ramsey in partial fulfillment of the requirements for the MA degree at the University of Dayton. The authors wish to acknowledge D. W. Biers, Department of Psychology, University of Dayton, for his contributions to the data analysis. Correspondence should be sent to D. J. Polzella, Department of Psychology, University of Dayton, 300 College Park, Dayton, OH 45469.
Nickerson (1965) favored the expectancy hypothesis, since he found that his subjects were increasingly likely to make presignal or unusually quick responses to the RS as the FP lengthened. He reasoned that to expect a signal to occur at a particular moment should not only facilitate response preparation, but also cause the subject sometimes to anticipate its occurrence by initiating a response before the signal is actually presented.

Ollman and Billington's (1972) deadline model represents a more elaborate and formal version of this hypothesis. According to their model, the subject sets a "deadline," which approximately coincides with the longest FP occurring within the experiment. Any RS that is presented before this point is simply detected and reacted to. As the deadline approaches, thus signalling the longest possible foreperiod, the subject uses this information to estimate the arrival of the RS and thereby times his or her response to coincide with its presentation. Ollman and Billington (1972) reported data that decisively support this model.

The implication of the results of experiments such as those of Nickerson (1965) and Ollman and Billington (1972) is that the foreperiod effect is partly an artifact of an anticipatory error rate that changes systematically as a function of the foreperiod duration. A question remains to be answered, however: What is the shape of the foreperiod effect when performance is errorless-that is, when the data reflect detection responses exclusively? The answer to this question is not immediately obvious, since most of the published reaction-time experiments, including those that have involved highly practiced subjects, have typically resulted in anticipatory error rates of $2 \%$ to 3\%. Yet Pachella (1974) has elegantly argued that, even when error rates are this low, the plotted functions may still reflect processes that cause errors.

The present experiment was designed to answer this question. Errors were discouraged in three ways: First, the subjects were instructed to trade off speed against accuracy and to adopt an errorless performance criterion 
Table 1

Probabilities of Anticipations, Probable Anticipations, and Overall Errors under No Catch-Trials and Catch-Trials Conditions

\begin{tabular}{llccccccc}
\hline & & & \multicolumn{7}{c}{ Probable Anticipations } \\
\cline { 3 - 7 } Subject & Condition & Anticipations & FP $_{1}$ & FP $_{2}$ & FP $_{3}$ & FP $_{4}$ & FP $_{5}$ & Overall \\
\hline D.W. & No catch & .002 & .00 & .00 & .00 & .00 & .01 & .004 \\
& Catch & .001 & .00 & .00 & .00 & .00 & .00 & .001 \\
P.M. & No catch & .000 & .00 & .00 & .00 & .01 & .01 & .005 \\
& Catch & .000 & .00 & .00 & .00 & .00 & .01 & .001 \\
D.P. & No catch & .000 & .00 & .00 & .00 & .00 & .01 & .003 \\
& Catch & .002 & .00 & .00 & .00 & .00 & .00 & .002 \\
E.R. & No catch & .002 & .00 & .00 & .01 & .01 & .01 & .009 \\
& Catch & .000 & .00 & .00 & .00 & .00 & .01 & .002 \\
Mean & No catch & .001 & .00 & .00 & .00 & .00 & .01 & .005 \\
& Catch & .001 & .00 & .00 & .00 & .00 & .00 & .002 \\
\hline
\end{tabular}

Note-Probable anticipations $=$ responses with RT $<130 \mathrm{msec}$. FP $1=300$-msec foreperiod; $\mathrm{FP}_{2}=400 \mathrm{msec}, \mathrm{FP}_{3}=500 \mathrm{msec}, \mathrm{FP}_{4}=600 \mathrm{msec}$, and $\mathrm{FP}_{5}=700 \mathrm{msec}$.

if possible. Second, the subjects received extensive practice, averaging over 450 trials at each of five foreperiods. Finally, the subjects were tested both with and without "catch trials," trials on which the RS did not follow the WS.

\section{METHOD}

\section{Subjects and Apparatus}

The subjects, who were volunteers, were three right-handed males (E.R., D.P., and P.M.) and one right-handed female (D.W.). They ranged in age from 22 to 40 years old. The apparatus consisted of a Commodore VIC-20 microcomputer, a Commodore model 1702 color monitor, and a Commodore model 1541 disk drive. The computer was programmed to control the presentation of warning signals, foreperiod durations, and response signals, as well as to time the subjects' responses and analyze the data. All timing subroutines were coded in 6502 machine language and adapted for the VIC-20 from Price's (1979) programs for the Apple II.

The WS consisted of two dots, $9 \mathrm{~mm}$ in diameter and $38 \mathrm{~mm}$ apart (horizontally), presented for approximately $100 \mathrm{msec}$ in the center of the $28.5 \times 1.5 \mathrm{~cm}$ CRT. Each FP was timed from the offset of the WS to the onset of the RS, which was a single dot, also $9 \mathrm{~mm}$ in diameter, presented at the center of the CRT. The average viewing distance was $40 \mathrm{~cm}$. The RS remained visible until the subject responded by pressing a function key located at the lower right-hand corner of the keyboard.

\section{Design and Procedure}

The experiment was conducted under two separate conditions, one that included catch trials and one that did not. Each condition consisted of a sequence of 10 blocks of 120 training trials, followed by a sequence of 10 blocks of 120 test trials. Within a block, each trial began with the presentation of the WS followed by one of five foreperiods: $\mathbf{3 0 0}$, $400,500,600$, or $700 \mathrm{msec}$. Each trial ended with the presentation (or nonpresentation, depending on the condition) of the RS. The particular foreperiod on any given trial was randomly determined. The intertrial interval was approximately $5 \mathrm{sec}$.

The subjects were instructed to respond quickly to the RS, but to attempt to achieve errorless performance-that is, to avoid anticipating the presentation of the RS. Under the no catch-trials condition an RS was presented with a probability of 1.00 ; under the catch-trials condition, an RS was presented with a probabily of 0.90 . For Subjects E.R., D.P., and P.M., the order of conditions consisted of catch trials followed by no catch trials. For Subject D.W., the order was reversed. Data were obtained from each subject over a period of approximately 3 weeks.

\section{RESULTS}

The error data, broken down by subject and condition, are summarized in Table 1. The table shows error rates for anticipations-responses that occurred either before the onset of RS or during a catch-trial interval-and for probable anticipations-responses in which the RT was so brief (less than $130 \mathrm{msec}$ ) that true detection of the RS was unlikely to have occurred (see Nickerson, 1965). Although none of the subjects achieved perfect performance, their anticipation rates were extremely low. Not surprisingly, the likelihood of an apparent anticipation was somewhat greater under the no catch-trials condition, but in no case did it exceed $1 \%$.

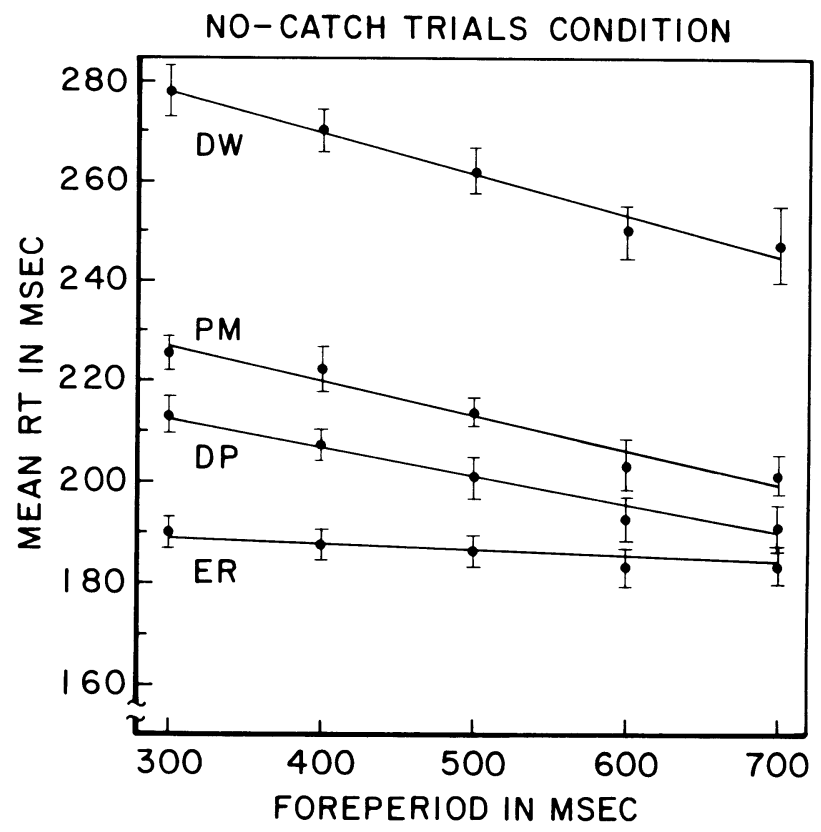

Figure 1. The effect of foreperiod duration on mean reaction time for the no catch-trials condition. Curves are best-fitting linear functions. Error bars are $\mathbf{9 9 \%}$ confidence intervals. 
CATCH TRIALS CONDITION

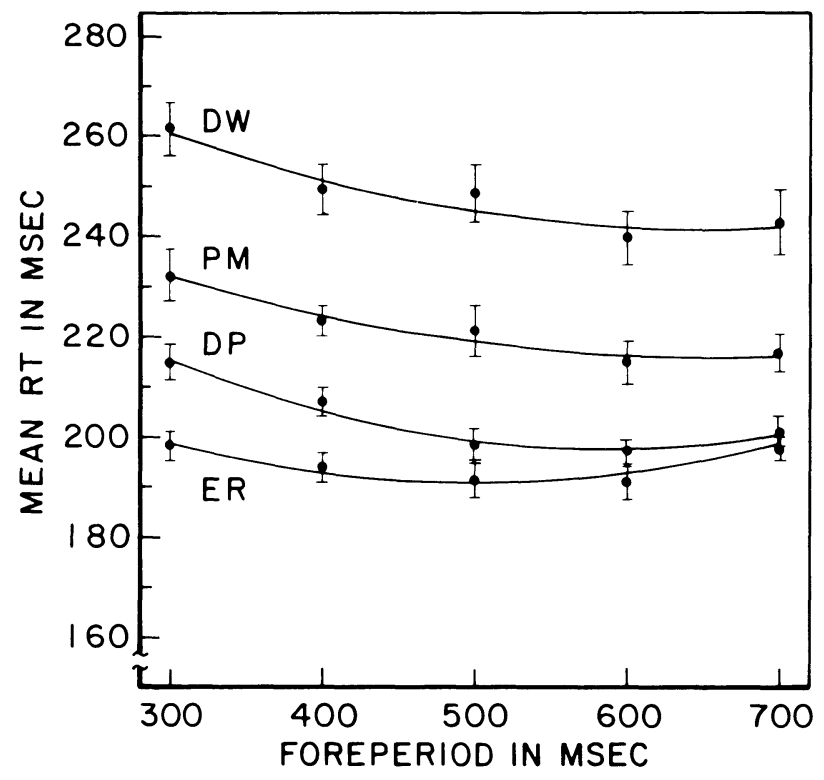

Figure 2. The effect of foreperiod duration on mean reaction time for the catch-trials condition. Curves are best-fitting second-order polynomial (quadratic) functions. Error bars are $99 \%$ confidence intervals.

Errorless RT functions are plotted in Figures 1 and 2 for the no catch-trials and catch-trials conditions, respectively. The curves are best-fitting linear functions in Figure 1 and best-fitting second-order polynomial (quadratic) functions in Figure 2. Table 2 reveals a statistical justification for plotting the data in this manner. The table shows the relative contribution of the linear and quadratic trend components to the overall treatment effect of the varying foreperiod duration. The measures, recommended by Keppel (1982, p. 140), are ratios of the component sum of squares to the treatment sum of squares. Table 2 reveals that there was a change in the errorless RT functions from linearity to quadratic curvilinearity, which reflected the shift from no catch trials to catch trials. Both sets of functions fit the data well and are consistent with the expectation that RT decreases as foreperiod lengthens.

\section{DISCUSSION}

It is well established that, for a uniform range of brief foreperiods, there is an inverse relationship between reaction time and foreperiod

Table 2

Relative Contribution of Linear and Quadratic Components to the Overall Treatment Effect of Foreperiod $\left(S S_{\text {trend }} / S S_{\text {treatment }}\right)$ for No Catch-Trials and Catch-Trials Conditions

\begin{tabular}{lccccc}
\hline & \multicolumn{2}{c}{ No catch Trials } & & \multicolumn{2}{c}{ Catch Trials } \\
\cline { 2 - 3 } \cline { 5 - 6 } Subject & Linear & Quadratic & & Linear & Quadratic \\
\hline D.W. & .99 & .00 & & .76 & .12 \\
P.M. & .92 & .00 & & .76 & .11 \\
D.P. & .91 & .04 & & .63 & .30 \\
E.R. & .49 & .30 & & .00 & .91 \\
Mean & .83 & .08 & & .54 & .36 \\
\hline
\end{tabular}

length. The present study reveals that this relationship still remains intact under relatively error-free performance. However, it could be argued that even though apparent anticipations were excluded from the plotted data, there is no assurance that all of the remaining responses were "detections." The data may still consist of a combination of responses, including anticipations that were not identified because they exceeded the 130 -msec criterion.

This argument is not compelling, however, for two reasons. First, the extremely low rate of anticipations suggests that, at most, a comparable rate of anticipations might have gone undetected. Even if they had occurred, these "undetected anticipations" would have been so rare as to have had a negligible effect on the plotted functions. Second, the subjects typically reported their being aware of having made an anticipation on a particular trial. In every case, we found that we could match these reports with the anticipations and probable anticipations that were subsequently identified. Thus, we are reasonably confident that the plotted functions reflect true detections exclusively.

The expectancy hypothesis, as it has been proposed by Nickerson (1965) and elaborated by Ollman and Billington (1972), is not fully consistent with the results of the present experiment. The hypothesis predicts that the decrease in reaction time as the foreperiod lengthens is due in part to the subject's being able to anticipate the response signal. But there was no evidence that the plotted functions were contaminated by errors of anticipation.

Perhaps the most plausible explanation of these findings would involve a modification of the expectancy hypothesis, in which reaction time could be said to covary with the momentary probability of occurrence of the response signal. It is assumed in the present situation that highly practiced subjects can and do learn to prepare to respond according to this momentary probability. In the no catch-trials condition, in which the momentary probability approaches 1.00 as the foreperiod lengthens, the subjects become increasingly certain that the response signal is imminent. This can be modeled reasonably well with a linear decrease in reaction time. In the catch-trials condition, where the momentary probability only approaches .90 as the foreperiod lengthens, the subjects become increasingly uncertain that a signal will occur at all. This can be modeled with a function in which reaction time decreases at first and then increases as a catch trial seems more likely.

If one assumes that both functions truly reflect errorless performance as we have argued, then an increase in the momentary probability of occurrence of the response signal will lead to an increase in the subject's readiness to detect that signal. Apparently, any concomitant tendency to anticipate can be successfully inhibited. This suggests that subjects in the present experiment adopted what Meyer, Yantis, Osman, and Smith (1984) call a "sensorial," as opposed to a "muscular," response strategy. Under this strategy, preparatory innervation is directed exclusively to the expected sense impression and not to the movement, which is inhibited until after the response signal is apprehended.

\section{REFERENCES}

KEPPEL, G. (1982). Design and analysis: A researcher's handbook (2nd ed.). Englewood Cliffs, NJ: Prentice-Hall.

Meyer, D. E., Yantis, S., Osman, A., \& Smith, J. E. K. (1984). Discrete and continuous models of response preparation: A reaction-time analysis. In S. Kornblum \& J. Requin (Eds.), Preparatory states \& processes (pp. 69-94). Hillsdale, NJ: Erlbaum.

Nickerson, R. S. (1965). Response time to the second of two successive signals as a function of absolute and relative duration of intersignal interval. Perceptual \& Motor Skills, 21, 3-10.

NiEmi, P., \& NAATANEN, R. (1981). Foreperiod and simple reaction time. Psychological Bulletin, 89, 133-162.

Ollman, R. T, \& Billington, M. J. (1972). The deadline model for simple reaction time. Cognitive Psychology, 3, 311-336.

Pachella, R. G. (1974). The interpretation of reaction time in information processing research. In B. H. Kantowitz (Ed.), Human information processing: Tutorials in performance and cognition (pp. 4182). Hillsdale, NJ: Erlbaum.

Price, J. M. (1979). Software timing for 6500 series microcomputers. Behavior Research Methods \& Instrumentation, 11, 568-571. 